\title{
The President's Page: Target Response or Target Dose
}

\author{
Jay N. Cohn
}

Published online: 28 May 2009

(C) Springer Science + Business Media, LLC 2009

A pharmacologic issue that has not received adequate attention is the appropriate dosing of cardiovascular drugs. Clinical investigators, biostatisticians and guideline authors have so muddied the water on this problem that they have confused the medical practice community. The International Society of Cardiovascular Pharmacotherapy owes it to our colleagues to deal with this confusion head-on.

Drugs such as antihypertensives and lipid-lowering agents have traditionally been dosed to achieve a target effect. In short-term clinical trials aimed at studying their blood pressure and LDL reducing properties, it has been standard practice to titrate the drugs to the desired target level of these so-called risk factors. This has been the advice promulgated in guidelines and advocated to practicing physicians.

When these drugs began to be used in other conditions and in long-term outcome trials, however, target responses were not always available. In heart failure, for instance, and in patients who have sustained a recent acute myocardial infarction, the use of these drugs was not to lower an elevated blood pressure or an elevated cholesterol but rather to favorably affect morbidity and mortality. Target dosing became the standard practice, and the dose selected was based on the dosages of the drug employed in the outcome trials. We now had a paradox. Should we be using target doses or target responses?

The goal of all cardiovascular therapy is to improve morbidity and mortality. Although popular surrogate markers such as blood pressure and cholesterol may

J. N. Cohn $(\bowtie)$

ISCP, University of Minnesota Medical School,

Minneapolis, MN 55455, USA

e-mail: cohnx001@umn.edu correlate with the risk of morbid events and with their prevention by therapy, it has become increasingly clear in recent years that the magnitude of benefit on the surrogate does not necessarily predict the benefit on outcome. Whether dealing with antihypertensive or lipid-lowering drugs of different classes it is apparent that the measured blood pressure reduction or LDL reduction are not necessarily the sole mechanism for the favorable effect of individual drugs.

In an effort to preserve the target response concept, advocates for these measures have fished in their data bases for what might be interpreted as the "optimal level" of these variables. These new levels, such as a blood pressure of $120 / 80 \mathrm{mmHg}$ or an LDL of $76 \mathrm{mg} / \mathrm{dl}$, have then emerged as the "target response". Unfortunately, however, these levels did not serve in the trials as the target response but rather were the observed response in the treated patients who benefited. An alternative hypothesis, of course, is that the drug produced the benefit through multiple mechanisms and that the response of the surrogate is in part a reflection of the favorable effect on the disease process or of a particularly responsive, low-risk patient.

Advocating target responses to primary care physicians is confusing. The recommended targets differ depending on the patient population, and physicians are notoriously reluctant to titrate drugs based on responses that are difficult to quantitate. Target dosing seems to be an easier recommendation. It has worked quite well in heart failure, for instance, where $\mathrm{ACE}$ inhibitor and ARB therapy recommendations have been based on dose.

The ISCP should foster a frank discussion of target response vs. target dose. Clarification of the use of these drugs could have a salutary effect on the international practice of medicine. 